\title{
Tipo de órbitas para una acción holomorfa afín en una variedad compleja
}

\author{
Tipe of orbits for holomorphic affine on a complex variety
}

\author{
Benito L. Ostos C.
}

\section{Resumen}

Dado una acción holomorfa afín $\varphi: \mathrm{A} f f(\mathrm{C}) \quad x \mathrm{M} \rightarrow \mathrm{M}$ del grupo $\mathrm{A} f f(\mathrm{C})$ del grupo sobre una variedad compleja $\mathrm{M}$. Se sabe que esta acción asocia dos campos holomorfos completos $X$ e $Y$ con $X$ periódica de periodo $2 \pi$ i y que están relacionados por el corchete de Lie mediante la relación. $[X, Y]=-\mathrm{Y}$. El conjunto singular de $\varphi$ es $\operatorname{sing}\left(\mathrm{Z}_{\varphi}\right)$ está dado por los puntos $p \in M$ tal que $Z_{\varphi}(p)=X(p) \wedge Y(p)=0$

Se resuelve el siguiente problema: En sing $\left(Z_{\varphi}\right)$ las órbitas de $\varphi$ son biholomorfas a un punto, a $\mathrm{C}_{\text {, a }} \mathrm{C}^{\wedge *} \mathrm{o}$ al toro complejo $T$ y en $M-\operatorname{sing}\left(Z_{\phi}\right)$ y en las órbitas son biholomorfas a $\mathbb{C}^{*} \times \mathbb{C}, \mathbb{C}^{*} \times \mathbb{C}^{*}, \mathbb{C}^{*} \times T$, o $T \times \mathbb{C}$.

Palabras claves: Acción holomorfa afín; campos holomorfos completos; corchete de Lie.

\begin{abstract}
Given holomorphic affine action $\varphi$ :Aff(C) $x \mathrm{M} \rightarrow \mathrm{M}$ of the group $\mathrm{A} f f(\mathrm{C})$ on a complex manifold $M$. It is known that this action associate two complete holomorphic fields $X$ and $Y$ with $X$ periodic of period and that related by the Lie bracket by the relation $[X, Y]=-Y$. The singular set of $\varphi$ it is $\operatorname{sing}\left(Z_{\varphi}\right)$ and this one given by the points $p \in M$ such th at $Z_{\varphi}(p)=X(p) \wedge Y(p)=0$.

The following problem is solved: In $\operatorname{sing}\left(\mathrm{Z}_{\varphi}\right)$ the orbits of $\varphi$ they are biholomorphic to a point, to $\mathrm{C}_{\text {, to }} \mathrm{C}^{*}$ or to the complex bull $T$ and in $\mathrm{M}-\operatorname{sing}\left(\mathrm{Z}_{\varphi}\right)$ the orbits are biholomorphic to $\mathrm{C}^{*} \mathrm{x} \mathrm{C}, \mathrm{C}^{*} \mathrm{x} \mathrm{C}^{*}, \mathrm{C}^{*} \mathrm{x} \mathrm{T}$, or $\mathrm{T} \times \mathrm{C}$.
\end{abstract}

Keywords: Action holomorphic affine; complete holomorphic fields; Lie bracket.

\section{Introducción}

Este artículo es dedicado al estudio de foliaciones holomorfas de codimensión uno en una variedad compleja de dimensión por lo menos tres, que provienen de acciones holomorfas afines. Una acción holomorfa afín es una acción holomorfa $\varphi$ :Aff(C) $\mathrm{x} \mathrm{M} \rightarrow \mathrm{M}$ del grupo $A f f(C)$ sobre una variedad compleja $M$ de dimensión $m$.

Se sabe que esta acción asocia dos campos holomorfos completos $X$ e $Y$ con $X$ periódica de periodo $2 \pi$ i y que están relacionados por el corchete de Lie mediante la relación $[X, Y]=-$ Y. Esto implica que podemos definir una foliación holomorfa de codimensión $m-2$. Se estudia los tipos de órbitas para ese tipo de acciones.

En esta dirección en el libro de César Camacho y Lins Neto (véase [1]) se puede encontrar una prueba para acciones afines reales y en la tesis de Rufo Cautinho (véase [3]) es para acciones holomorfas afines sobre una variedad de Stein $M$.

La contribución que se hace en este trabajo es cuando $M$ es una variedad compleja cualquiera no necesariamente es de Stein.

El presente trabajo de investigación tiene los siguientes objetivos:
- Si $p \in \operatorname{sing}\left(Z_{\varphi}\right)$ entonces la órbita de la acción $\varphi$ que pasa por el punto $p, O_{p}(\varphi)$, es isomorfa analítica a $\{\mathrm{p}\}$ o C o $\mathrm{C}^{*}$ al toro complejo $T$.

- Las órbitas en $M-\operatorname{sing}\left(Z_{\omega}\right)$ de la acción holomorfa afín $\varphi$ son biholomorfas a $\mathbb{C}^{*} x \mathbb{C} \circ \mathbb{C}^{*} x \mathbb{C}^{*} \circ \mathbb{C}^{*} x T \circ T \times \mathbb{C}$.

\section{Materiales y métodos}

Preliminares

En esta sección se enuncian las definiciones y propiedades que se usarán a lo largo del trabajo.

\section{Acciones holomorfas y campos vectoriales}

Sea $\mathrm{G}$ un grupo de Lie complejo con una operación * y $M$ una variedad compleja. Decimos que $\varphi: G \times M \rightarrow \mathrm{M}$ es una acción holomorfa del grupo $\mathrm{G}$ sobre la variedad $M$ cuando $\varphi$ es un mapeo holomorfo y satisface las siguientes condiciones:

1. $\varphi(e, p)=p$ para todo $p \in M$, donde e es la identidad de $\mathrm{G}$.

2. $\varphi\left(g_{1} * g_{2}, p\right)=\varphi\left(g_{1}, \varphi\left(g_{2}, p\right)\right)$, para todo $g_{1}, g_{1} \in G \mathrm{y}$ para todo $p \in M$.

El inverso de cualquier element $g \in G$ es denotado por $\mathrm{g}^{-1}$. El grupo de isotropía, la órbita y el conjunto de puntos fijos de esta acción es denotado y definido por $G_{p}(\varphi)=\{g \in G: \varphi(g, p)=p\}, \mathcal{O}_{p}(\varphi)=\{\varphi(g, p): g \in G\}$ y $\Sigma(\varphi)=$ $\{p \in M: \varphi(g, p)=p$, para todo, $t \in \mathbb{C}\}$ respectivamente. 
En $\mathrm{G}$ se define la relación de equivalencia $\sim$ dado por: $g_{1} \sim g_{2} \Leftrightarrow g_{1}^{-1} * g_{2} \in G_{p}(\varphi)$ para todo $g_{1}, g_{2} \in G$. La clase de equivalencia de un punto $g \in G$ es denotado por $[g]$ y el conjunto cociente $G / G_{p}=\{|g|: g \in G\}$ es un grupo de Lie complejo. La aplicación $\tilde{\varphi}_{x}: \frac{G}{G} \rightarrow \mathcal{O}_{p}(\varphi)$, definido por $\tilde{\varphi}_{x}[g]=\varphi_{x}(g)$ es un biholomorfismo entre las variedades $G / G p$ y $O p(\varphi)$ (para estos resultados véase [4] ).

Cuando $\left(\mathrm{G},{ }^{*}\right)=(\mathrm{C},+)$ se obtiene un campo holomorfo completo $X$ definido como su generador infinitesimal, es decir, para cada $p \in M$ se tiene

$X(p)=\left.\frac{\partial}{\partial t} \varphi(t, p)\right|_{t=0}$. Lo recíproco también es cierto, es decir: si $X$ es un campo holomorfo completo en $M$ se puede asociar una acción holomorfa de C sobre $M$. Si $p \in M-\sum \quad$ se sabe que $G_{\mathrm{p}}=Z w_{1}+Z w_{2} \quad$ y $O_{p}(\varphi)$ es isomorfo analítico al grupo cociente $C / G_{\mathrm{p}}$ y que a su vez es isomorfo a una de las superficies de Riemann $\mathrm{C}, \mathrm{C}^{*} \mathrm{o}$ al toro complejo $T$ de dimensión uno. A partir de aquí se denota $X_{t}(p)=\varphi(t, p)$.

Definición 1 Sean $M$ y $N$ variedades complejas, $X, Y$ dos campos vectoriales sobre $M$ y $N$. Si $p$ y $q$ son dos puntos de $\mathrm{M}$ y $N$ respectivamente, se dice que son localmente conjugados en $p$ y $q$ si existen dos conjuntos abiertos $U \subset M, V \subset N, \mathrm{P} \in \mathrm{U}, q \in V \quad$ y un biholomorfismo $\Phi: \mathrm{U} \rightarrow \mathrm{V}, \Phi(p)=q$ tal que $D \Phi \circ\left(\left.X\right|_{U}\right)=\left.Y\right|_{V} \circ \Phi$. Teorema 1 (Teorema del flujo tubular) Sea $X$ un campo vectorial sobre $\mathrm{M}$ y un punto $p \in M$ tal que $\mathrm{X}(p) \neq 0$. Entonces $X$ es localmente conjugada en $p$ y $0 \in \mathbb{C}^{m}$ al campo constante $\frac{\partial}{\partial z_{1}}$.

Para la prueba véase [8].

Definición 2 Sean $M$ y $N$ variedades complejas y $f: N \rightarrow M$ un biholomorfis-mo. Si $X$ es un campo vectorial holomorfo en $M$, se define un campo vectorial holomorfo $f^{*}(X)$ en $\mathrm{N}$ por $f^{*}(X)(p)=\mathrm{Df}^{-1}(f(p)) . X(f(p))$, para todo $p \in N$.

Sea $X$ e $Y$ dos campos holomorfos en $M$. Fijando un punto $p \in M$ y otro punto $t \in \mathbb{R}$, el vector

$$
v(t)=X_{t}^{*}(Y)(p)=D X_{-t}\left(X_{t}(p)\right) \cdot Y\left(X_{t}(p)\right)
$$

es tangente a $M$ en $p$.

Definición 3 El corchete de Lie de $X$ e $Y$ es un campo vectorial $[X, Y]$ definido por

$$
[X, Y](p)=\left.\frac{d}{d t}\left(X_{t}^{*}(Y)(p)\right)\right|_{t=0}, \quad \quad p \in M
$$

Lema 1 Sea $z: U \subset M \rightarrow \mathbb{C}^{m}$ una carta local. En esta carta local se escribe $X=\sum_{i=1}^{m} a_{i} \frac{\partial}{\partial z_{i}}$ e $Y \sum_{i=1}^{m} b_{i} \frac{\partial}{\partial z_{i}}$ Entonces $[X, Y]=\sum_{i=1}^{m} c_{i} \frac{\partial}{\partial z_{i}}$, donde

$$
c_{i}=\sum_{j=1}^{m}\left(a_{j} \frac{\partial b_{i}}{\partial z_{j}}-b_{j} \frac{\partial a_{i}}{\partial z_{j}}\right), i=1,2, \ldots, m .
$$

Para la prueba véase [1].

\section{Acción holomorfa afín}

Sea el grupo afín denotado como $A f f(C)$, , es decir, el conjunto de los automorfismos de $\mathrm{C}$ en $\mathrm{C}$, este es un grupo no abeliano con la composición de funciones afines. Ahora sea el grupo no abeliano $\mathrm{C}^{*} x \mathrm{C}$ con la operación $(a, b) \circ\left(a^{\prime}, b^{\prime}\right):=\left(a a^{\prime}, a b^{\prime}+b\right)$.
Los dos grupos anteriores son isomorfos y en adelante se considerará que ambos grupos son indistinguibles.

Definición 4 Una acción holomorfa $\varphi$ :Aff(C) x $M \rightarrow M$ del grupo $A f f(\mathrm{C})$ sobre una variedad compleja $M$ de dimensión por lo menos tres es llamada acción holomorfa afín.

Por la operación o del grupo $\mathrm{C}^{*} x \mathrm{C}$ se tiene:

$\varphi_{(s, t)}=\varphi_{(1, t) \circ(s, 0)}=\varphi_{(1, t)}{ }^{\circ}(s, 0)$, para todo $(s, t) \in \mathbb{C}^{*} \times \mathbb{C}$ lo cual nos permite asociar dos acciones una en $\mathbb{C}$ y la otra en $\mathbb{C}^{*}$ sobre $\mathrm{M}$ :

$$
\psi: \mathbb{C}^{*} \times M \rightarrow M y \zeta: \mathbb{C} \times M \rightarrow M
$$

$(s, p) \mapsto \varphi_{(s, 0)}(p)(t, p) \mapsto \varphi_{(1, t)}(p)$

Sea $\tilde{\psi}: \mathbb{C} \times M \rightarrow M$ definido como $\tilde{\psi}_{t}(p)=\varphi_{\left(e^{t}, 0\right)}(p)$ para todo $(t, p) \mathbb{C} \times M$. Se puede probar de manera trivial las siguientes propiedades:

1. $\tilde{\psi}$ es una acción holomorfa periódica de periodo $2 \pi \mathrm{i}$, esto es para cada $t \in \mathbb{C}$ se cumple $\tilde{\psi}_{t+2 \pi i}=\tilde{\psi}_{t}$

$$
\begin{aligned}
& \text { 2. } \tilde{\psi}_{t}(p)=\psi_{\left(e^{t}\right)}(p) \\
& \text { 3. } e^{G_{p}(\tilde{\psi})}=G_{p}(\psi) \\
& \text { 4. } \sum(\varphi)=\sum(\psi) \cap \sum(\zeta)
\end{aligned}
$$

Definición 5 La acción $\varphi$ es localmente libre si los grupos de isotropía $\mathrm{G}_{p}(\varphi)$ son discretos. La acción es foliada si todas las órbitas $\mathrm{O} p(\varphi)$ tienen la misma dimensión. Se denota por $Z_{\varphi}=X \wedge Y$ la sección global del fibrado $\wedge^{2} T^{\prime} M=T^{\prime} M \wedge T^{\prime} M \quad$ ( $T^{\prime} M$ es el fibrado tangente holomorfo), donde $X$ e $Y$ son los campos asociados a los flujos $\psi$ y $\zeta$ respectivamente. Usando el hecho de que $X(p) \wedge Y(p)=0$ si y solo si existe un tal $k \in \mathbb{C}$ que, se tiene que para cada punto en $M$ fuera del conjunto analítico $\operatorname{sing}\left(Z_{\varphi}\right)=\left\{p \in M: Z_{\varphi}(p)=0\right\}$ los campos $X \mathrm{e}$ $Y$ son linealmente independientes y esto es equivalente a que $\varphi$ sea localmente libre en cada punto de $\mathrm{M}-\operatorname{sing}(\mathrm{Z} \varphi)$ (véase [3,pp. 13]).Sea $F^{0}(\varphi)$ la foliación definido por las órbitas $\varphi$ en $\mathrm{M}-\operatorname{sing}(\mathrm{Z} \varphi)$.

Los campos $X$ e $Y$ están relacionados por el corchete de Lie $[X, Y]-Y$. Esto se prueba de la siguiente manera

$$
\begin{aligned}
& \begin{aligned}
X_{s}^{*}(Y)(p) & =\left.\left.\frac{d}{d t}\left(X_{-s} \circ Y_{t} \circ X_{s}\right)(p)\right|_{t=0} \frac{d}{d t}\left(\varphi_{\left(e^{-s, 0)}\right.} \circ \varphi_{(1, t)} \circ \varphi_{\left(e^{s}, 0\right)}(p)\right)\right|_{t=0} \\
& =\left.\frac{d}{d t} \varphi_{\left(1, e^{-s} t\right)}(p)\right|_{t=0}=\left.e^{-s} \frac{d}{d t} \varphi_{(1, t)}(p)\right|_{t=0}=e^{-s} Y(p) .
\end{aligned} \\
& \text { Luego } \\
& {[X, Y](p)=\left.\frac{d}{d s} X_{S}^{*}(Y)(p)\right|_{s=0}=-\left.e^{-s} Y(p)\right|_{s=0}=-Y(p)}
\end{aligned}
$$

\section{METODOLOGÍA}

El conjunto sing $(Z \varphi)$ es un conjunto analítico de codimensión mayor o igual

a uno. Fuera de este conjunto se tiene una foliación holomorfa $\mathrm{F} 0(\varphi)$ de codimensión $\operatorname{dim} M-2$ dados por las órbitas de la acción. Para cada $p \in \operatorname{sing}\left(Z_{\varphi}\right)$ las órbitas son:

1. Si $X(p)=Y(p)=0$ entonces $\mathcal{O}_{p}(\varphi)=p$

2. Si $X(p)=0$ e $Y(p) \neq 0$ entonces $\mathcal{O}_{p}(\varphi)$ es isomorfo a una de las superficies de Riemann: $\mathbb{C}^{*}, \mathbb{C}$ o al toro complejo $\mathrm{T}$.

3. $\mathrm{S} X(p) \neq 0$ e $Y(p)=0$ entonces $\mathcal{O}_{p}(\varphi)$ es isomorfo 
analítico a una de las superficies de Riemann: $\mathbb{C}^{*}$ o al otro complejo $T$.

4. Si $X(p) \neq 0, Y(p) \neq 0 \quad$ y son linealmente dependientes, se obtiene la misma conclusión que el caso anterior

Para cada $p \in M-\operatorname{sing}\left(Z_{\varphi}\right)$ el grupo de isotropía es un grupo discreto y se estudia las relaciones que hay entre este grupo y los grupos de isotropía de las acciones que provienen de los campos $X$ (es decir, $\left.G_{p}(\psi)\right)$ e Y (es decir, $\left.G_{p}(\zeta)\right)$

Los resultados previos al respecto están descritos en el Lema 2 y 3 . El grupo de isotropía $G_{p}(\zeta)$ y $G_{p}(\psi)$ son de la forma:

1. $\{0\}, \lambda_{1} \mathbb{Z} \circ \lambda_{2} \mathbb{Z}+\lambda_{3} \mathbb{Z}$, donde $\lambda_{1} \in \mathbb{C}^{*} \mathrm{y} \lambda_{2}, \lambda_{3} \in \mathbb{C}$ son $\mathbb{R}$-linealmente independientes.

2. $\{1\} \circ \beta_{1}^{\mathbb{Z}}$, donde $\beta_{1}$ es de módulo $\neq 0$ y 1 , o raíz de la unidad, respectivamente.

Teniendo en cuenta estos lemas se analiza $G_{p}(\varphi)$. Para ello se fija un punto $(\mathrm{a}, \mathrm{b})$ en el grupo de isotropía $G_{p}(\varphi)$ :

1. Si $a \neq 1$ con $|a|>1 o|a|<1$ entonces $G_{p}(\varphi)$ es isomorfo a $\{(1,0)\}, \quad \beta_{1}^{\mathbb{Z}} \times\{0\}, \quad \beta_{2}^{\mathbb{Z}} \times\{0\}, \quad \beta_{3}^{\mathbb{Z}} \beta_{4}^{\mathbb{Z}} \times\{0\}, \quad$ donde $\beta_{1}, \beta_{2}, \beta_{3} y \beta_{4}$ son como en (3).

2. Si $a \neq 1$ con $|a|=1$. Si existe algún punto (c,d) $\in G_{p}(\varphi)$ con $|c|>1 o|c|<1$ es como en el caso 1 , en caso contrario $G_{p}(\varphi) \subset\left\{(c, d) \in S^{1} \times \mathbb{C}: c\right.$ es raíz de la unidad $\} \subset S^{1} \times \mathbb{C}$

De esto se obtiene que es isomorfo a

$\beta_{2}^{\mathbb{Z}} \times\{0\}, \quad(-1)^{\mathbb{Z}} \times \lambda_{1} \mathbb{Z}(-1)^{\mathbb{Z}} x\left(\lambda_{2} \mathbb{Z}+\lambda_{3} \mathbb{Z}\right)$.

Si $a=1$ y fijemos un punto $(c, d) \in G_{p}(\varphi)$ Si $c \neq 1$ hacer lo mismo que en los casos anteriores. Si $c=1$.

Sea $\left(a^{\prime}, b^{\prime}\right) \in G_{p}(\varphi)$. Si $\left|a^{\prime}\right|>1 o\left|a^{\prime}\right|<1$ se llega una contradicción. Si $a^{\prime} \neq 1$ con $\left|a^{\prime}\right|=1$ estamos como en el caso 2 . Si $a^{\prime} \neq 1$ se tiene entonces que

$G_{p}(\varphi) \subset L=\{(1, y): y \in \mathbb{C}\} \quad$ y de esto $G_{p}(\varphi)$ es isomorfo a $\{(1,0)\},\{1\} \times \lambda_{1} \mathbb{Z},\{1\} \times \lambda_{2} \mathbb{Z}+\lambda_{3} \mathbb{Z}$

Ya que la órbita de la acción holomorfa afín en el punto $p, \mathcal{O}_{p}(\varphi)$ es isomorfo analítico a la variedad compleja $\mathbb{C}^{*} x \mathbb{C} / G_{p}(\varphi)$ se obtiene el Teorema 3 .

\section{Resultados y discusión}

Los resultados de la investigación están dados en las teoremas 2 y 3 de esta sección.

Teorema 2 Sea $\varphi$ es una acción holomorfa afín sobre una variedad compleja $M$.

Si $p \in \operatorname{sing}\left(Z_{\varphi}\right)$ entonces $\mathcal{O}_{p}(\varphi)$ es isomorfa analítica a a $\{p\}$ o $\mathbb{C}$ o $\mathbb{C}^{*}$ o al toro complejo $T$.

Demostración: Teniendo en cuenta $\psi$ y $\zeta$ dado en (1) se procede a la prueba.

Si $X(p)=0 e Y(p)=0$ entonces $O_{p}(\varphi)=\{p\}$.

$$
\begin{aligned}
& \text { Si } X(p)=0 \text { e } Y(p) \neq 0 \text { entonces } \\
& \mathcal{O}_{p}(\varphi)=\left\{\varphi_{(s, t)}(p):(s, t) \in \mathbb{C}^{*} x \mathbb{C}\right\} \\
& =\left\{\varphi_{(1, t)} \circ \varphi_{(s, 0)}(p):(s, t) \in \mathbb{C}^{*} x \mathbb{C}\right\} \\
& =\left\{\varphi_{(1, t)}(p): t \in \mathbb{C}\right\} \\
& =\mathcal{O}_{p}(\zeta)
\end{aligned}
$$

y esto es isomorfo a $\mathbb{C}$ o a $\mathbb{C}^{*}$ o a $T$.

Si $X(p) \neq 0$ e $Y(p)=0$ se define una aplicación $F: \mathcal{O}_{p}(\varphi) \rightarrow \mathcal{O}_{p}(\psi)$ como $F\left(\varphi_{(s, t)}(p)\right)=\varphi_{(s, 0)}(p)$ para todo $(s, t) \in \mathbb{C}^{*} x \mathbb{C}$.

Sea $F\left(\varphi_{(s, t)}(p)\right)=F\left(\varphi_{\left(s^{\prime}, t^{\prime}\right)}(p)\right)$, entonces $\varphi_{\left(s^{\prime} / s, 0\right)}(p)=p$. Luego se tiene

$$
\begin{aligned}
\varphi_{(s, t)^{-1} \circ\left(s^{\prime}, t^{\prime}\right)}(p) & =\varphi_{(1 / s,-t / s) \circ\left(s^{\prime}, t^{\prime}\right)}(p)=\varphi_{\left(s^{\prime} / s,\left(t^{\prime}-t\right) / s\right)}(p) \\
& =\varphi_{\left(1,\left(t^{\prime}-t\right) / s\right)_{\circ}\left(s^{\prime} / s, 0\right)}(p)=\varphi_{\left(1,\left(t^{\prime}-t\right) / s\right)^{0}} \varphi_{\left(s^{\prime} / s, 0\right)}(p) \\
& =\varphi_{\left(1,\left(t^{\prime}-t\right) / s\right)}(p)=p,
\end{aligned}
$$

esto es, $\varphi_{(s, t)}(p)=\varphi_{\left(s^{\prime}, t^{\prime}\right)}(p) \quad$ lo cual prueba que $\mathrm{F}$ es inyectiva. La sobreyectividad es evidente. Por lo tanto, $\mathcal{O}_{p}(\varphi)$ es isomorfa analítica a $\mathcal{O}_{p}(\psi)$ y esto es isomorfa a $\mathbb{C}^{*}$ o a $T$.

Si $X(p) \neq 0$ e $Y(p) \neq 0$, entonces como $X(p)$ e $Y(p)$ son linealmente dependientes y el grupo de isotropía $G_{p}(\varphi)$ no es discreto. Luego la dimensión de $\mathcal{O}_{p}(\varphi)$ es igual a uno, lo cual significa que $\mathcal{O}_{p}(\varphi)$ es isomorfa a $\mathcal{O}_{p}(\zeta)=\mathcal{O}_{p}(\psi)$ y esto es isomorfa a $\mathbb{C}^{*}$ o a $T$.

Proposición 1 Sea $\varphi$ una acción holomorfa afín sobre una variedad compleja $M$ y sea $p \in M-\operatorname{sing}\left(Z_{\varphi}\right)$, entonces $\varphi$ es conjugado analítico en una vecindad de $p$ a una acción $\varphi_{(s, t)}^{0}=Y_{t}^{0} \circ X_{l n s}^{0}$, donde $X_{s}^{0}$ e $Y_{t}^{0}$ son acciones en una variedad de cero cuyos campos asociados son

$\left.X^{0}=\dot{[} z_{1}+h\left(\dot{z}_{2}, \ldots, z_{m}\right)\right] \frac{\partial}{\partial z_{1}}+\sum_{j=2}^{m} a_{j}\left(z_{2}, \ldots, z_{m}\right) \frac{\partial}{\partial z_{j}}$ e $Y^{0}=\frac{\partial}{\partial z_{1}}$ respectivamente. Aquí $a_{j}(0) \neq 0$ para algún $j \in\{2,3, \ldots, m\}$

Demostración: Como $Y(p) \neq 0$ entonces por el Teorema del Flujo Tubular se puede hacer un cambio de coordenadas en una vecindad de $p$ tal que $Y=\frac{\partial}{\partial z_{1}}$ y en este sistema de coordenadas se escribe

$$
X=\sum_{j=1}^{m} a_{j}\left(z_{1}, \ldots, z_{m}\right) \frac{\partial}{\partial z_{j}}
$$

Usando $[X, Y]=-Y$ se tiene

$$
\sum_{j=1}^{m}\left(-\frac{\partial a_{j}}{\partial z_{1}}\right) \frac{\partial}{\partial z_{j}}=-\frac{\partial}{\partial z_{1}}
$$

Esto da $\mathrm{a}_{1}=\mathrm{z}_{1}+\mathrm{h}\left(\mathrm{z}_{2}, \ldots, \mathrm{z}_{\mathrm{m}}\right)$ y las funciones $\mathrm{a}_{2}, \ldots, \mathrm{a}_{\mathrm{m}}$ no dependen de la variable $z_{1}$. Luego $X$ es,

$X=\left[z_{1}+h\left(z_{2}, \ldots, z_{m}\right)\right] \frac{\partial}{\partial z_{1}}+\sum_{j=2}^{m} a_{j}\left(z_{2}, \ldots, z_{m}\right) \frac{\partial}{\partial z_{j}}$

Como en este sistema de coordenadas $X(0) \neq 0, Y(0) \neq 0$ y además son linealmente independientes entonces $a_{j}(0) \neq 0$ para algún $j \in\{2,3, \ldots, m\}$.

Definición 6 Un punto $p \in M$ es un punto regular por $\varphi$ si $\varphi$ si $Z_{\varphi}(p) \neq 0$ o existe una función holomorfa $h_{p}$ definido en una vecindad de $p$ con $\mathrm{h}_{\mathrm{p}}(\mathrm{p})=0$ y tal que $\frac{1}{h_{p}} Z_{\varphi}$ es holomorfo y no cero en $p$. Un punto $p \in M$ es llamado un punto singular de $\varphi$ si no es un punto regular de $\varphi$.

El conjunto de puntos singulares de $\varphi$ es denotado por $\operatorname{sing}(\varphi)$. Por la definición anterior, $\operatorname{sing}(\varphi) \subset \operatorname{sing}\left(Z_{\varphi}\right)$ y por $M$. Suzuki [9] $\operatorname{sing}(\varphi)$ es un subconjunto analítico de $M$ de codimensión mayor o igual a 2. Por un argumento de Hartogs (véase la Proposición 11 de [5]) se puede extender la foliación $F^{0}(\varphi) \mathrm{M}$-sing $\left(\mathrm{Z}_{\varphi}\right)$ a una foliación en $M-\operatorname{sing}(\varphi)$ lo cual denotaremos por $F(\varphi)$.

\section{Algunos ejemplos}


La foliación $F(\varphi)$ puede tener hojas contenidas dentro del conjunto $\operatorname{sing}\left(Z_{\varphi}\right)$. El siguiente ejemplo muestra que $\operatorname{sing}(\varphi)$ está contenido estrictamente en $\operatorname{sing}\left(Z_{\varphi}\right)$ y no tiene hojas dentro de $\operatorname{sing}\left(Z_{\varphi}\right)-\operatorname{sing}(\varphi)$.

\section{Ejemplo 1 Sea}

$X\left(z_{1}, z_{2}, z_{3}\right)=z_{1} \frac{\partial}{\partial z_{1}}-2 z_{2} \frac{\partial}{\partial z_{2}}-2 z_{3} \frac{\partial}{\partial z_{3}}$ e $Y\left(z_{1}, z_{2}, z_{3}\right)=$ $\left(z_{2}+z_{3}+z_{1}^{2}\right) \frac{\partial}{\partial z_{1}}+\left(z_{1} z_{2}+z_{1} z_{3}+z_{1}^{3}\right) \frac{\partial}{\partial z_{2}}+\left(z_{1} z_{2}+z_{1} z_{3}+z_{1}^{3}\right) \frac{\partial}{\partial z_{3}}$ dos campos holomorfos en $\mathbb{C}^{3}$. Se tiene que $X(0)=Y(0)=0$ y el corchete de Lie $[X, Y]=-Y$ entonces podemos definir una acción afín en $\mathbb{C}^{3}$ que denotaremos que $\varphi$ y que es localmente libre en $\mathbb{C}^{3}-\operatorname{sing}\left(Z_{\varphi}\right)$. Veamos cual es este conjunto singular.

$X \wedge Y\left(z_{1}, z_{2}, z_{3}\right)=\left(z_{2}+z_{3}+z_{1}^{2}\right)\left[\left(-z_{1}^{2}+2 z_{2}\right) \frac{\partial}{\partial z_{1}} \wedge \frac{\partial}{\partial z_{2}}+\left(-z_{1}^{2}+2 z_{3}\right) \frac{\partial}{\partial z_{1}} \wedge \frac{\partial}{\partial z_{3}}\right.$ Se tiene que

$$
\left.+2 z_{1}\left(-z_{2}+z_{3}\right) \frac{\partial}{\partial z_{2}} \wedge \frac{\partial}{\partial z_{3}}\right]
$$

$\operatorname{sing}\left(Z_{\varphi}\right)=\left\{\left(z_{1}, z_{2}, z_{3}\right): z_{2}+z_{3}+z_{1}^{2}=0\right\} \cup\left\{\left(z_{1}, z_{2}, z_{3}\right): z_{1}^{2}-2 z_{2}=0, z_{2}-\right.$ $\left.z_{3}=0\right\}$

El primero es un conjunto analítico de codimensión uno y el segundo es un conjunto analítico de codimensión 2 que viene ser $\operatorname{sing}(\varphi)$. El conjunto $\left\{\left(z_{1}, z_{2}, z_{3}\right): z_{2}+z_{3}+z_{1}^{2}=0\right\}$ está contenido en $\operatorname{sing}\left(Z_{\varphi}\right)$ y no es invariante por la foliación $F(\varphi)$.

Ejemplo 2 Sea una acción afín $\varphi: A f f(\mathbb{C}) \times \mathbb{C}^{3} \rightarrow \mathbb{C}^{3}$ definido por

$\varphi_{(\mathrm{s}, \mathrm{t})}\left(\mathrm{z}_{1}, \mathrm{z}_{2}, \mathrm{z}_{3}\right)=\left(\mathrm{t}+\mathrm{sz}_{1}+1-\mathrm{s}, \mathrm{s}^{\lambda_{2}} \mathrm{z}_{2}, \mathrm{~s}^{\lambda_{3}} \mathrm{z}_{3}\right)$, donde $\lambda_{2}, \lambda_{3} \in \mathbb{Z}$.

Los campos $X$ e $Y$ asociados a esta acción son:

$X\left(z_{1}, z_{2}, z_{3}\right)=\left(z_{1}-1\right) \frac{\partial}{\partial z_{1}}+\lambda_{2} z_{2} \frac{\partial}{\partial z_{2}}+\lambda_{3} z_{3} \frac{\partial}{\partial z_{3}}$ e $Y\left(z_{1}, z_{2}, z_{3}\right)=\frac{\partial}{\partial z_{1}}$ Como se puede ver $X(0,0,0) \neq 0, \quad Y(0,0,0) \neq 0$ y X e Y son linealmente dependientes en $(0,0,0)$. En este ejemplo $z_{\varphi}\left(z_{1}, z_{2}, z_{3}\right)=X\left(z_{1}, z_{2}, z_{3}\right) \wedge Y\left(z_{1}, z_{2}, z_{3}\right)=$ $-\lambda_{2} z_{2} \frac{\partial}{\partial z_{1}} \wedge \frac{\partial}{\partial z_{2}}-\lambda_{3} z_{3} \frac{\partial}{\partial z_{1}} \wedge \frac{\partial}{\partial z_{3}}$

de esto se tiene que $(0,0,0) \in \operatorname{sing}(\varphi)$.

\section{Tipo de órbitas}

Sea $\varphi: A f f(\mathbb{C}) x M \rightarrow M$ una acción afín en una variedad compleja $M$ de dimensión por lo menos 3. Cuando $p \in M-\operatorname{sing}\left(Z_{\varphi}\right)$ el grupo de isotropía $\mathrm{G}_{n}(\varphi)$ es un subgrupo discreto de $\mathbb{C}^{*} \boldsymbol{x} \mathbb{C}$. De ahí $G_{p}(\psi)^{n} y G_{p}(\zeta)$ es isomorfo a $\left\{(s, 0): \varphi_{(s, 0)}(p)=p\right\} \subset G_{p}(\varphi) y\left\{(1, t): \varphi_{(1, t)}(p)=p\right\} \subset G_{p}(\varphi)$ respectivamente. Luego el grupo de isotropía $G_{p}(\psi)$ un subgrupo discreto de $\mathbb{C}^{*}$ y $G_{p}(\zeta)$ es un subgrupo discreto de $\mathbb{C}$. Además el grupo de isotropía () también es discreto en $\mathbb{C}$.

Por un lado se sabe que los grupos discretos de $\mathbb{C}$ son de la forma:

$\{0\}, \lambda_{1} \mathbb{Z} y \quad \lambda_{2} \mathbb{Z}+\lambda_{3} \mathbb{Z}$,

donde $\lambda_{1} \in \mathbb{C}^{*}$ y $\lambda_{2}, \lambda_{3} \in \mathbb{C} \quad$ son $\mathbb{R}$ linealmente independientes. Por otro lado los grupos discretos $\mathbb{C}^{*}$ de están dados por (véase [6,pp,5]:

$\{1\}, \beta_{1}^{\mathbb{Z}}, \beta_{2}^{\mathbb{Z}}$ y $\beta_{3}^{\mathbb{Z}} \beta_{4}^{\mathbb{Z}}$, donde $\beta_{1}, \beta_{3}$ son números complejos de módulo distintos de cero y de uno mientras que $\beta_{2}, \beta_{4}$ son números complejos con raíz de la unidad. Aquí $\beta_{1}^{\mathbb{Z}}:=\left\{\beta_{i}^{n}: n \in \mathbb{Z}\right\}$.

Es claro que $G_{p}(\psi) \times G_{p}(\zeta)$ es un subconjunto pero no necesariamente es un subgrupo de $G_{p}(\varphi)$. Al tomar el producto cartesiano de $G_{p}(\psi)$ con $G_{p}(\zeta)$ se tiene grupos discretos en $\left(\mathbb{C}^{*} x \mathbb{C}, 0\right)$ :

1. $\{(1,0)\},\{1\} \times \lambda_{1} \mathbb{Z},\{1\} \times\left(\lambda_{2} \mathbb{Z}+\lambda_{3} \mathbb{Z}\right)$,

2. $\beta_{1}^{\mathbb{Z}} \times\{0\}, \beta_{1}^{\mathbb{Z}} \times \lambda_{1} \mathbb{Z}$ con $\beta_{1} \in \mathbb{Z}, \beta_{1}^{\mathbb{Z}} \times\left(\lambda_{2} \mathbb{Z}+\lambda_{3} \mathbb{Z}\right) \operatorname{con} \beta_{1} \in \mathbb{Z}$,

3. $\beta_{2}^{\mathbb{Z}} \times\{0\},(-1)^{\mathbb{Z}} \times \lambda_{1} \mathbb{Z},(-1)^{\mathbb{Z}} \times\left(\lambda_{2} \mathbb{Z}+\lambda_{3} \mathbb{Z}\right)$,

4. $\beta_{3}^{\mathbb{Z}} \beta_{4}^{\mathbb{Z}} \times\{0\}, \beta_{3}^{\mathbb{Z}}(-1)^{\mathbb{Z}} \times \lambda_{1} \mathbb{Z}$ con $\beta_{3} \in$

$\mathbb{Z}, \beta_{3}^{\mathbb{Z}}(-1)^{\mathbb{Z}} \times\left(\lambda_{3} \mathbb{Z}+\lambda_{4} \mathbb{Z}\right)$ con $\beta_{3} \in \mathbb{Z}$.

Lema 2 El grupo de isotropía $G_{p}(\zeta)$ y $G_{p}(\psi)$ son de las formas:

1. $\{0\}, \lambda_{1} \mathbb{Z}, \lambda_{2} \mathbb{Z}+\lambda_{3} \mathbb{Z}$, donde $\lambda_{1} \in \mathbb{C}^{*}$ y $\lambda_{2}, \lambda_{3} \in \mathbb{C}$ son $\mathbb{R}$-linealmente independientes.

2. $\{1\}, \beta_{1}^{\mathbb{Z}}$, donde $\beta_{1}$ es de módulo $\neq 0$ y 1 , o raíz de la unidad, respectivamente.

Demostración: La primera afirmación es claro ya que $G_{p}(\zeta)$ es un subgrupo discreto cerrado de $\mathbb{C}$.

Como $\tilde{\psi}$ es un flujo periódico de periodo $2 \pi$ i se tiene que $2 \pi i \mathbb{Z} \subset G_{p}(\tilde{\psi})$ entonces $G_{p}(\tilde{\psi})$ es de la forma

$2 \pi i \mathbb{Z} \circ 2 \pi i \mathbb{Z}+\beta \mathbb{Z}$,

donde $2 \pi i \mathbb{Z}$ y $\beta$ son $\mathbb{R}$-linealmente independientes.

La otra opción posible es $G_{p}(\tilde{\psi})=\gamma_{1} \mathbb{Z}+\gamma_{2} \mathbb{Z}$, donde $\gamma_{1} \mathrm{y}$ $\gamma_{2}$ ambos distintos de $2 \pi i \mathbb{Z}$ pero $\mathbb{R}$-linealmente independiente. Usando $e^{G_{p}(\tilde{\psi})}=G_{p}(\psi)$ y este último es discreto en $\mathbb{C}^{*}$ tiene que $\left|e^{\gamma_{1}}\right| \neq 1$ y $e^{\gamma_{2}}$ raíz de la unidad, es decir, existe un $k \in N$ tal que $e^{k \gamma_{2}}=1$. Como $2 \pi i \in G_{p}(\tilde{\psi}) \quad$ entonces $2 \pi i=\gamma_{1} m+\gamma_{2} n$, donde $m, n \in \mathbb{Z}$. Multiplicando por k luego elevando la exponencial tendremos

$$
(1,0)=e^{2 \pi i k}=e^{\gamma_{1} k m} e^{\gamma_{2} k n}=e^{\gamma_{1} k m}
$$

Ahora aplicando módulo $1=\left|e^{\gamma_{1}}\right|^{m k} \quad$ esto es una contradicción. Nuevamente usando $e^{G_{p}(\psi)}=G_{p}(\psi)$ en (4) se tiene el resultado deseado.

Lema 3 Si $G_{p}(\psi)=\beta_{1}^{\mathbb{Z}}$, donde $\beta_{1}$ es de módulo $\neq 0$ y 1 , o raíz de la unidad entonces el grupo cociente $\mathbb{C}^{*} / G_{p}(\tilde{\psi})$ es isomorfo analítico al toro complejo $T$.

Demostración: Primero tenemos que el grupo de isotropía $G_{p}(\tilde{\psi})=2 \pi i \mathbb{Z}+\beta \mathbb{Z}$, donde $\mathrm{e}^{\beta}=\beta_{1}$. Se sabe además que $\mathbb{C} / G_{p}(\tilde{\psi})$ es isomorfo analítico al toro complejo $T$. Luego el isomorfismo es el siguiente

$$
\begin{aligned}
f: \mathbb{C} / G_{p}(\tilde{\psi}) & \rightarrow \mathbb{C}^{*} / G_{p}(\psi) \\
t+G_{p}(\tilde{\psi}) & \mapsto e^{t} G_{p}(\psi) .
\end{aligned}
$$

La función $f$ está bien definido: Sea $\mathrm{t}_{1} \mathrm{y}_{2}$ dos elementos en $\mathbb{C}$ tales

que $-t_{1}+t_{2} \in G_{p}(\tilde{\psi})$ entonces $e^{-t_{1+} t_{2}} \in G_{p}(\psi)$ y esto implica que

$f\left(t_{1}+G_{p}(\tilde{\psi})\right)=f\left(t_{2}+G_{p}(\tilde{\psi})\right)$.

La función $f$ es inyectiva: Sea $f\left(t+G_{p}(\tilde{\psi})\right)=G_{p}(\tilde{\psi})$ entonces por definición de $f$ tendremos $e^{t} G_{v}(\psi)=G_{v}(\psi)$ y esto es

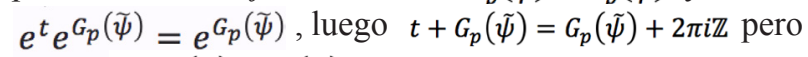
esto es $t+G_{p}(\tilde{\psi})=G_{p}(\tilde{\psi})$. 
En el siguiente teorema se prueba los tipos de órbitas que se encuentran fuera del conjunto $\operatorname{sing}(Z \varphi)$, para ello se usa la misma técnica dada en la Proposición 4 de [1,pp. 171].

Teorema 3 Las órbitas en $M-\operatorname{sing}(Z \varphi)$ de la acción holomorfa afín $\varphi$ son biholomorfas a

\section{$\mathbb{C}^{*} x \mathbb{C} \circ \mathbb{C}^{*} x \mathbb{C}^{*} \circ \mathbb{C}^{*} x T \circ T \times \mathbb{C}$.}

Demostración: Sea $H=G p(\varphi)$ este es un subgrupo discreto cerrado de $\mathbb{C}^{*} x \mathbb{C}$ (aquí $p$ esta fuera del conjunto analítico $\sin g(Z \varphi))$. Fijemos un punto $(a, b) \in H$.

Caso 1. Si $a \neq 1$ con $|a|>1$ ㅇa $\mid<1$

Como $a \neq 1$ entonces $(a, b)^{n}=\left(a^{n}, b\left(a^{n}-1\right)(a-1)^{-1}\right)$, para todo $n \in \mathbb{Z}$. Fijando otro punto $(c, d) \in H$ se tiene

$h_{n}=(a, b)^{-n} \circ(c, d) \circ(a, b)^{n}=\left(c, \frac{1}{a-1}\left[b c\left(1-a^{-n}\right)+b\left(a^{-n}-1\right)\right]+d a^{-n}\right)$ Si $|a|>1$ (en el caso de que $|a|<1$ tomar $(\mathrm{a}, \mathrm{b})^{-1}=\left(\mathrm{a}^{-1},-\mathrm{b} / a\right)$ y hacer el mismo análisis) entonces

$\lim _{n \rightarrow \infty} h_{n}=(c,(b c-b) /(a-1)=h \in H)$. Por ser $H$ discreto se tiene que $h_{n}=h$ para todo $n \geq n_{0}$ lo que implica que $(a, b) \circ(c, d)=(c, d) \circ(a, b)$ esto es $b(c-1)=d(a-1)$. De todo esto obtendremos que

$H \subset L=\{(x, y) \in \mathbb{C} x \mathbb{C}: b(x-1)=(a-1) y\}$ Sea $f: \mathbb{C}^{*} x\{0\} \rightarrow L \cap \mathbb{C}^{*} x \mathbb{C}$ definido como $f(s, 0)=(s, k(s-1))$, donde $\mathrm{k}=\mathrm{b} /(\mathrm{a}-1)$. Es claro que $f$ es un isomorfismo entre estos dos grupos. Entonces $\mathrm{f}^{-1}(\mathrm{H})$ será una de las siguientes formas $\{(1,0)\}, \beta_{1}^{\mathbb{Z}} \times\{0\}, \beta_{2}^{\mathbb{Z}} \times\{0\}, \beta_{3}^{\mathbb{Z}} \beta_{4}^{\mathbb{Z}} \times\{0\}$, donde $\beta_{1}, \beta_{2}, \beta_{3}$ y $\beta_{4}$ son como en (3). El último caso no se da debido a que

$G_{p}(\varphi)=\left\{(s, 0) \in \mathbb{C}^{*} x \mathbb{C}: \varphi_{(s, 0)}(p)=p\right\}=G_{p}(\psi) \times\{0\}$ es isomorfo a $\beta_{2}^{\mathbb{Z}} \beta_{3}^{\mathbb{Z}}$ y esto es una contradicción con el Lema 3.

Caso 2. Si $\mathrm{a} \neq 1$ con $|\mathrm{a}|=1$ (de esto se tiene que la sucesión $\left(a^{n}\right)_{n \geq 1}$ no es convergente). Si existe un punto $(c, d) \in H$ con $|c|>10|c|<1$ entonces es como en el caso 1 .

Veamos como es H cuando la primera componente de sus puntos está en el círculo unitario. El conjunto

$<(a, b)\rangle:=\left\{(a, b)^{n}=\left(a^{n}, b\left(a^{n}-1\right) /(a-1)\right): n \in \mathbb{Z}\right\}$ está contenido en el cilindro $S^{1} \times \mathbb{C}$ y es un subgrupo de $H$. Si $a$ no es raíz de la unidad entonces

$\overline{\langle(a, b)\rangle}=L \cap\left(S^{1} x \mathbb{C}\right)$ donde $L=\{(x, y) \in \mathbb{C} x \mathbb{C}: b(x-1)=(a-1) y\}$ Como $H$ es un conjunto cerrado se tiene que $L \cap\left(S^{1} x \mathbb{C}\right) \subset H$, esto produce una contradicción con el hecho de que $H$ es discreto. Por lo tanto $a$ es raíz de la unidad y esto implica que $\langle(a, b)>$ es un conjunto finito contenido en $H$. Por lo tanto:

$H \subset\left\{(c, d) \in S^{1} x \mathbb{C}: c\right.$ es raíz de la unidad $\} \subset S^{1} x \mathbb{C}$.

Sea

$$
\begin{aligned}
f: \mathbb{C}^{*} \times\{0\} & \rightarrow L \cap \mathbb{C}^{*} \times \mathbb{C} \\
(s, 0) & \mapsto(s, k(s-1))
\end{aligned}
$$

donde $\mathrm{k}=\mathrm{b} /(\mathrm{a}-1)$. Esta aplicación es biyectiva. Como $<(a, b)>$ es un subgrupo de $H$ entonces $\left.f^{-1}(<(a, b)\rangle\right)$ es un subgrupo de $\mathrm{S}^{1}$. Sea $\pi: \mathbb{C}^{*} x \mathbb{C} \rightarrow \mathbb{C}^{*}$ dado por $\pi(s, t)=s$. Todas las imágenes recíprocas, $\pi^{-1}\left(a^{n}\right) \cap H$, para cada son isomorfas a un único subgrupo discreto de $\mathbb{C}$. Por lo tanto
$H$ es isomorfa a uno de los siguientes grupos:

$\beta_{2}^{\mathbb{Z}} \times\{0\},(-1)^{\mathbb{Z}} \times \lambda_{1} \mathbb{Z},(-1)^{\mathbb{Z}} \times\left(\lambda_{2} \mathbb{Z}+\lambda_{3} \mathbb{Z}\right)$ donde $\beta_{2}$ es raíz de la unidad $\lambda_{1} \in \mathbb{C}^{*} \mathrm{y} \lambda_{2}, \lambda_{3}{ }^{\prime}$ números complejos $\mathbb{R}$-linealmente independientes.

Caso 3. Si a $=1$ y se fija un punto $(c, d) \in H$. Si $\mathrm{c} \neq 1$ se sigue los pasos como en los casos anteriores. Si $\mathrm{c}=1$, sea $\left(a^{\prime}, b^{\prime}\right) \in H$ entonces

$h_{n}=\left(a^{\prime}, b^{\prime}\right)^{-n} \circ(1, b) \circ\left(a^{\prime}, b^{\prime}\right)^{n}=\left(1, b a^{\prime-n}\right)$

Si $\left|a^{\prime}\right|>1$ tomando el límite $\lim _{n \rightarrow \infty} h_{n}=(1,0)$ y como $H$ discreto se tiene que a partir de un $n$ grande $\left(1, b a^{\prime-n}\right)=(1,0)$ y esto implica $a^{\prime}=0$ produciendo una contradicción. Se tiene el mismo resultado cuando $\left|a^{\prime}\right|>1$. Si $a^{\prime} \neq 1$ con $\left|a^{\prime}\right|=1$ estamos como en el caso 2. Si $\left|a^{\prime}\right|=1$ se tiene entonces que $H \subset L=\{(1, y): y \in \mathbb{C}\}$,

es claro que $L$ es isomorfo a $\mathbb{C}$, por lo tanto $H$ toma uno de las siguientes formas:

$\{(1,0)\},\{1\} \times \lambda_{1} \mathbb{Z},\{1\} \times \lambda_{2} \mathbb{Z}+\lambda_{3} \mathbb{Z}$

Luego la variedad compleja $\mathbb{C}^{*} x \mathbb{C} / G_{p}(\varphi)$ tiene las siguientes formas posibles:

1. $\mathbb{C}^{*} x \mathbb{C} /\{(1,0)\} \approx \mathbb{C}^{*} x \mathbb{C}$

2. $\mathbb{C}^{*} x \mathbb{C} /\left(\{1\} x \lambda_{1} \mathbb{Z}\right) \approx \mathbb{C}^{*} x \mathbb{C}^{*}$

3. $\mathbb{C}^{*} \times \mathbb{C} /\left(\{1\} x \lambda_{2} \mathbb{Z}+\lambda_{3} \mathbb{Z}\right) \approx \mathbb{C}^{*} \times T$

4. $\mathbb{C}^{*} \times \mathbb{C} / \beta_{1}^{\mathbb{Z}} \times\{0\} \approx T \times \mathbb{C}$

5. $\mathbb{C}^{*} x \mathbb{C} / \beta_{2}^{\mathbb{Z}} x\{0\} \approx \mathbb{C}^{*} x \mathbb{C}$

6. $\mathbb{C}^{*} x \mathbb{C} /\left((-1)^{\mathbb{Z}} x\left\{\lambda_{1} \mathbb{Z}\right\}\right) \approx \mathbb{C}^{*} x \mathbb{C}^{*}$

7. $\mathbb{C}^{*} x \mathbb{C} /\left((-1)^{\mathbb{Z}} x\left(\left\{\lambda_{2} \mathbb{Z}+\lambda_{3} \mathbb{Z}\right\}\right)\right) \approx \mathbb{C}^{*} x T$

8. $\mathbb{C}^{*} \times \mathbb{C} / \beta_{3}^{\mathbb{Z}} \beta_{4}^{\mathbb{Z}} \times\{0\} \approx T \times \mathbb{C}$,

donde $\lambda_{1}, \lambda_{2}, \lambda_{3}, \beta_{1}, \beta_{2} y \beta_{3}$ omo en (2) y (3). Además el símbolo $\approx$ indica isomorfismo analítico entre ambos conjuntos.

Por lo tanto la órbita de que pasa por el punto $p$ es isomorfo a una de las siguientes formas posibles

$\mathbb{C}^{*} \times \mathbb{C}, \mathbb{C}^{*} \times \mathbb{C}^{*}, \mathbb{C}^{*} \times T, T \times \mathbb{C}$, donde $T$ es el toro complejo de dimensión uno.

A partir de aquí se construyen explícitamente estos isomorfismos analíticos.

Para $\mathbb{C}^{*} x \mathbb{C} /\left(\{1\} x \lambda_{1} \mathbb{Z}\right) \approx \mathbb{C}^{*} x \mathbb{C}^{*}$ se considera la aplicación

$$
\begin{aligned}
& F: \mathbb{C}^{*} \times \mathbb{C} /\{1\} \times \lambda \mathbb{Z} \rightarrow \mathbb{C}^{*} \times(\mathbb{C} / \lambda \mathbb{Z}) \\
& (s, t) \quad \mapsto \quad(s,[t / s]) .
\end{aligned}
$$

a) F está bien definido

Sea $\left(s^{\prime}, t^{\prime}\right) \in[(s, t)]$ es decir que

$\left.(s, t)^{-1} \circ\left(s^{\prime}, t^{\prime}\right)=\left(s^{\prime} / s,\left(t^{\prime}-t\right) / s\right) \in G_{p}(\varphi)=[1] x \lambda \mathbb{Z}\right)$ entonces existe $m \in \mathbb{Z}$ tal que $\left(s^{\prime} / s,\left(t^{\prime}-t\right) / s\right)=(1, \lambda m)$ comparando términos $s^{\prime}=s y t^{\prime} / s^{\prime}-t / s=\lambda m$ y esto implica que $t^{\prime} / s^{\prime} \in[s, t]$

b) $F$ es inyectiva

Sea $F([(s, t)])=F\left(\left[\left(s^{\prime}, t^{\prime}\right)\right]\right)$ entonces $s=s^{\prime}$ y $[t / s]=\left[t^{\prime} / s^{\prime}\right]$ de esta última igualdad existe $m \in \mathbb{Z}$ tal que

$t^{\prime} / s^{\prime}-t / s=\lambda m$ esto implica que $t^{\prime}-t=\lambda m s \quad$ y esto significa que $[(s, t)]=\left[\left(s^{\prime}, t^{\prime}\right)\right]$. 
c) F es sobreyectiva

Sea $(s,[\tilde{t}]) \in \mathbb{C}^{*} x(\mathbb{C} / \lambda \mathbb{Z})$, tomando $t=\tilde{t} s+\lambda m s$ se tiene que $F(s, t)=(s,[t / s])=(s,[\tilde{t}+\lambda m])=(s,[\tilde{t}])$. Por lo tanto la órbita de $\varphi$ es isomorfo a $\mathbb{C}^{*} x(\mathbb{C} / \lambda \mathbb{Z})$, esto es isomorfo a $\mathbb{C}^{*} \boldsymbol{x} \mathbb{C}^{*}$.

Para $\mathbb{C}^{*} x \mathbb{C} / \beta_{2}^{\mathbb{Z}} x\{0\} \approx \mathbb{C}^{*} x \mathbb{C}$. Como $\beta_{2}$ es raíz de la unidad entonces se puede escribir $\mathrm{e}^{2 \pi \mathrm{i} / \mathrm{q}}=\beta_{2}$, donde $p, q \in \mathbb{Z}$ con $\mathrm{q}>0$ Se define la siguiente aplicación

$$
\begin{aligned}
\mathbb{C} / \frac{2 \pi i p}{q} \mathbb{Z} & \rightarrow \mathbb{C}^{*} / \beta_{2}^{\mathbb{Z}} \\
{[t]_{\mathbb{C}} } & \mapsto\left[e^{t}\right]_{\mathbb{C}^{*}}
\end{aligned}
$$

y se prueba que es una biyección.

Para $\mathbb{C}^{*} x \mathbb{C} / \beta_{3}^{\mathbb{Z}} \beta_{4}^{\mathbb{Z}} \times\{0\} \approx T \times \mathbb{C}$. Ya que $\beta_{3}^{\mathbb{N}} \beta_{4}^{\mathbb{N}} \operatorname{con} \beta_{3}$ de módulo distinto de cero, uno y $\beta_{4}$ es raíz de la unidad se tiene $e^{\alpha}=\beta_{3}$ y $e^{2 \pi i p / q}=\beta_{4}$ con $\alpha \in \mathbb{C}$ y $p, q \in \mathbb{Z}$ con $q>0$. Definiendo la aplicación

$$
\begin{aligned}
\mathbb{C} /\left(\alpha \mathbb{Z}+\frac{2 \pi i p}{q} \mathbb{Z}\right) & \rightarrow \mathbb{C}^{*} / \beta_{3}^{\mathbb{Z}} \beta_{4}^{\mathbb{Z}} \\
{[t]_{\mathbb{C}} } & \mapsto\left[e^{t}\right]_{\mathbb{C}^{*}}
\end{aligned}
$$

es otra biyección. Luego $\mathbb{C}^{*} / \beta_{2}^{\mathbb{Z}}, \mathbb{C}^{*} / \beta_{3}^{\mathbb{Z}} \beta_{4}^{\mathbb{Z}}$ es isomorfo a $\mathbb{C}^{*}$ y a $T$, respectivamente.

Cuando $p \in M-\operatorname{sing}\left(Z_{\varphi}\right)$ entonces por la Proposición 1 la acción holomorfa afín $\varphi$ es conjugada analítica a $\varphi^{0}$ en una vecindad de $p$. La acción holomorfa afín $\varphi^{0}$ esta dado por dos campos holomorfos:

$\left.X^{0}\left(z_{1},{ }^{\prime} z\right)=\left(z_{1}, h{ }^{\prime} z\right)\right) \frac{\partial}{\partial z_{1}}+\sum_{j=2}^{m} a_{j}\left({ }^{\prime} z\right) \frac{\partial}{\partial z_{j}^{\prime}}$ con $a_{j}\left({ }^{\prime} 0\right) \neq 0$ para algún $j=2, \ldots, m$ y por $Y^{0}\left(z_{1},{ }^{\prime} z\right)=\frac{\partial}{\partial z_{1}}$. A partir de esto se tiene

$\varphi_{(s, t)}^{0}(z)=\left(t+\varphi_{(s, 0)}^{01}(z), \varphi_{(s, 0)}^{02}(z), \ldots, \varphi_{(s, 0)}^{0 m}(z)\right)$ para todo $(s, t)$ en una vecindad abierta $V$ de $(1,0)$ en $\tau^{* *} \boldsymbol{x}($. Aquí $\varphi_{(s, 0)}^{0 j}(0) \neq 0$ para al menos un $j=2, \ldots, m$ y para $s$ en una vecindad de 1 en $\mathbb{C}^{*}$.

Ejemplo 3 Sea $\varphi: \mathbb{C}^{*} x \mathbb{C} x \mathbb{C}^{m} \rightarrow \mathbb{C}^{m}$ una acción afín holomorfo definido por

$\varphi_{(s, t)}(z)=\left(t-k+\left(z_{1}+k\right) s, s^{\lambda_{2}} z_{2}, \ldots, s^{\lambda} m_{m}\right)$ donde $k \in \mathbb{C}$ y los $\lambda_{j}{ }^{\prime} s$ pertenecen a $\mathbb{Z}$ con al menos algún $\lambda_{j} \neq 0$. Como

$Z_{\varphi}(z)=\lambda_{2} z_{2} \frac{\partial}{\partial z_{2}} \wedge \frac{\partial}{\partial z_{1}}+\lambda_{3} z_{3} \frac{\partial}{\partial z_{3}} \wedge \frac{\partial}{\partial z_{1}}+\cdots+\lambda_{m} \lambda_{m} \frac{\partial}{\partial z_{m}} \wedge \frac{\partial}{\partial z_{1}}$

Se tiene $\operatorname{sing}\left(z_{\varphi}\right)=\left\{\left(z_{1}, 0, \ldots, 0\right): z_{1} \in \mathbb{C}\right\}$. El grupo de isotropía es $G_{z}(\varphi)=\left\{(s, t) \in \mathbb{C}^{*} x \mathbb{C}: t-k+\left(z_{1}+k\right) s=z_{1}, s^{\lambda} j z_{j}=z_{j}\right.$ para todo $j=2, \ldots, m\}$. Si $\mathrm{Z}=\left(\mathrm{z}_{1}, 0, \ldots, 0\right)$ entonces $G_{z}(\varphi) \subset L-\left\{\left(0, z_{1}+k\right)\right\}$, donde $L=\left\{t-k+\left(z_{1}+\right.\right.$ $\left.k) s=z_{1}\right\}$; esto implica que es isomorfo a un subgrupo discreto de $\mathbb{C}^{*}$. Si $\mathrm{z}=\left(\mathrm{z}_{1}, \ldots, \mathrm{z}_{\mathrm{m}}\right)$ con algún $z_{j} \neq 0$ para $j \in\{2,3, \ldots m\}$ entonces $s^{\lambda_{j}}=1$. Por lo tanto $\mathrm{G}_{\mathrm{z}}(\varphi)$ es un conjunto finito isomorfo a $\beta_{2}^{\mathbb{Z}}$, para algún $\beta_{2}$ raíz de la unidad.

Esta misma acción se puede extender a una acción holomorfa afín en el espacio proyectivo complejo $\mathbb{C} P(m)$ de dimensión $m$. Sea $U_{0}=\left\{\left[z_{0}: z_{1}: \ldots: z_{m}\right]: z_{0} \neq 0\right\}$ y el biholomorfismo $\emptyset_{0}: U_{0} \rightarrow \mathbb{C}^{m}$ definido como $\emptyset_{0}\left(\left[z_{0}: z_{1}: \ldots: z_{m}\right]\right)=\left(z_{1} / z_{0}, z_{2} / z_{0}, \ldots, z_{m} / z_{0}\right)$. Ahora consideremos la composición $\emptyset_{0}^{-1} \circ \varphi_{(s, t)} \circ \emptyset_{0}\left(\left[z_{0}: z_{1}: \ldots: z_{m}\right]\right)={ }^{\prime}\left[1: t-k+\left(\frac{z_{1}}{z_{0}}+k\right) s: s^{\lambda_{2}} \frac{z_{2}}{z_{0}}: \ldots: s^{\lambda_{m}} \frac{z_{m}}{z_{0}}\right]$ $=\left[z_{0}:(t-k) z_{0}+\left(z_{1}+k z_{0}\right) s: s^{\lambda_{2}} z_{2}: \ldots: s^{\lambda m} z_{m}\right]$.

Sea $\tilde{\varphi}: \mathbb{C}^{*} x \mathbb{C} x \mathbb{C} P^{m} \rightarrow \mathbb{C} P^{m}$ definido por $\widetilde{\varphi}\left(\left[z_{0}: z_{1}: \ldots: z_{m}\right]\right)=\left[z_{0}:(t-k) z_{0}+\left(z_{1}+k z_{0}\right) s: s^{\lambda_{2}} z_{2}: \ldots: s^{\lambda_{m}} z_{m}\right]$ Se prueba sin mayor dificultad que es una acción holomorfa afín bien definida en $\mathbb{C} P^{m}$

\section{Conclusiones}

En $\operatorname{sing}\left(Z_{\varphi}\right)$ las órbitas de son biholomorfas a un punto, a $\mathbb{C}, \mathbb{C}^{*}$ o al toro complejo $T$; mientras que $M-\operatorname{sing}\left(Z_{\varphi}\right)$ las órbitas son biholomorfas a $\mathbb{C}^{*} \times \mathbb{C}, \mathbb{C}^{*}, \mathbb{C}^{*} x T \circ a T \times \mathbb{C}$.

\section{Literatura citada}

C. Camacho y A. Lins Neto. 1979: Teoría geométrica das folheações; Impa.

E.M. Chirka 1989. Complex analytic sets; Matematics and its Aplications (Soviet Series), Vol. 46, Kluwer Academia Publishers.

R. Coutinho: Linearization of affine group actions; Rio de Janeiro, (2008).

Dimitri, N. Akhiezer. 1997. Lie Group Actions in Complex Analysis, Aspects of Mathematics, Moscow.

Lins, N. y Scárdua, B. 1997. Fotlheações algébricas complexas, Impa.

A. Poirier 2005. Aspectos geométricos del análisis complejo, Pontificia Universidad Católica del Perú, Fondo Editorial.

Rebelo, J.C 1996. Singularités des flots holomorphes, Ann. De I'Institute Fourier 46: 411- 428.

Sotomayor, JM. 1978. Lições de equações diferenciais ordinárias, Instituto de Matemática Pura y Aplicada (Proyecto Euclides).

S. Suzuki, 1977. Sur les opérations holomorphes de et de sur un space de Stein: Séminaire Norguet, Springer Lect. Notes, 670, 80-88. 